Full length article

\title{
Reinforcement of poly-L-lactic acid electrospun membranes with strontium borosilicate bioactive glasses for bone tissue engineering
}

\author{
João S. Fernandes ${ }^{\mathrm{a}, \mathrm{b}}$, Piergiorgio Gentile ${ }^{\mathrm{c}}$, Margarida Martins ${ }^{\mathrm{a}, \mathrm{b}}$, Nuno M. Neves ${ }^{\mathrm{a}, \mathrm{b}}$, Cheryl Miller ${ }^{\mathrm{c}}$, \\ Aileen Crawford ${ }^{\mathrm{c}}$, Ricardo A. Pires ${ }^{\mathrm{a}, \mathrm{b}, *}$, Paul Hatton ${ }^{\mathrm{c}, *}$, Rui L. Reis ${ }^{\mathrm{a}, \mathrm{b}}$ \\ a 3B's Research Group - Biomaterials, Biodegradables and Biomimetics, University of Minho, Headquarters of the European Institute of Excellence on Tissue Engineering \\ and Regenerative Medicine, AvePark, 4806-909 Taipas, Guimarães, Portugal \\ b ICVS/3B's - PT Government Associate Laboratory, Braga/Guimarães, Portugal \\ ${ }^{\mathrm{c}}$ Centre for Biomaterials and Tissue Engineering, School of Clinical Dentistry, University of Sheffield, Claremont Crescent, Sheffield S10 2TA, United Kingdom
}

\section{A R T I C L E I N F O}

\section{Article history:}

Received 7 June 2016

Received in revised form 8 August 2016

Accepted 19 August 2016

Available online 21 August 2016

\section{Keywords:}

Strontium borosilicate glasses

PLLA membranes

Mineralisation

Osteogenic differentiation

BM-MSCs

\begin{abstract}
A B S T R A C T
Herein, for the first time, we combined poly-L-lactic acid (PLLA) with a strontium borosilicate bioactive glass (BBG-Sr) using electrospinning to fabricate a composite bioactive PLLA membrane loaded with $10 \%(\mathrm{w} / \mathrm{w})$ of BBG-Sr glass particles (PLLA-BBG-Sr). The composites were characterised by scanning electron microscopy (SEM) and microcomputer tomography ( $\mu$-CT), and the results showed that we successfully fabricated smooth and uniform fibres (1-3 $\mu \mathrm{m}$ in width) with a homogeneous distribution of BBG-Sr microparticles $(<45 \mu \mathrm{m})$. Degradation studies (in phosphate buffered saline) demonstrated that the incorporation of BBG-Sr glass particles into the PLLA membranes increased their degradability and water uptake with a continuous release of cations. The addition of BBG-Sr glass particles enhanced the membrane's mechanical properties (69\% higher Young modulus and 36\% higher tensile strength). Furthermore, cellular in vitro evaluation using bone marrow-derived mesenchymal stem cells (BM-MSCs) demonstrated that PLLA-BBG-Sr membranes promoted the osteogenic differentiation of the cells as demonstrated by increased alkaline phosphatase activity and up-regulated osteogenic gene expression (Alpl, $S p 7$ and Bglap) in relation to PLLA alone. These results strongly suggest that the composite PLLA membranes reinforced with the BBG-Sr glass particles have potential as an effective biomaterial capable of promoting bone regeneration.
\end{abstract}

\section{Statement of Significance}

PLLA membranes were reinforced with $10 \%(\mathrm{w} / \mathrm{w})$ of strontium-bioactive borosilicate glass microparticles, and their capacity to induce the osteogenic differentiation of bone marrow mesenchymal stem cells (BM-MSCs) was evaluated. These membranes presented an increased: degradability, water uptake, Young modulus and tensile strength. We also demonstrated that these membranes are non-cytotoxic and promote the attachment of BM-MSCs. The addition of the glass microparticles into the PLLA membranes promoted the increase of ALP activity (under osteogenic conditions), as well as the BM-MSCs osteogenic differentiation as shown by the upregulation of Alpl, Sp7 and Bglap gene expression. Overall, we demonstrated that the reinforcement of PLLA with glass microparticles results in a biomaterial with the appropriate properties for the regeneration of bone tissue.

(c) 2016 Acta Materialia Inc. Published by Elsevier Ltd. All rights reserved.

\footnotetext{
* Corresponding authors at: 3B's Research Group - Biomaterials, Biodegradables and Biomimetics, University of Minho, Headquarters of the European Institute of Excellence on Tissue Engineering and Regenerative Medicine, AvePark, 4806-909 Taipas, Guimarães, Portugal (R.A. Pires).

E-mail addresses: rpires@dep.uminho.pt (R.A. Pires),paul.hatton@sheffield.ac.uk (P. Hatton).
}

\section{Introduction}

The development of biomaterials for guided bone tissue engineering (BTE) has been a hot topic of research in recent years. Biomaterials can act as a short-term template in which cells proliferate and deposit extracellular matrix (ECM), helping bone ingrowth as a part of its regeneration process. A suitable biomaterial for BTE should present appropriate mechanical properties that 
are able to withstand the mechanical stress occurring at the lesion site and to support cell ingrowth as part of the bone formation process. The capacity to shape the biomaterial substrate to fit exactly in the bone lesion is of particular relevance, too [1,2].

Electrospinning is a versatile technique that applies electrostatic forces to manufacture ultrathin fibre meshes from melted polymers or polymer solutions. This methodology has been used to obtain biomaterials composed by micro- or nano-fibres with high surface areas and mechanical properties suitable for BTE by playing with its different processing parameters [2,3]. Furthermore, electrospun membranes closely mimic the ECM promoting cell attachment and proliferation [4].

Poly-L-lactic acid (PLLA) is a biodegradable and biocompatible polymer, widely investigated for BTE, specially due to the approval of different PLLA medical devices for clinical use by the US Federal Food and Drug Administration (FDA) [5]. Furthermore, PLLA has appropriate flexibility and deformation capacity, and can be processed by different techniques (e.g. melt, dry and wet spinning, as well as electrospinning) [6-9]. However, PLLA is not commonly considered osteoinductive $[4,10]$. Thus, in order to improve the biological performance of PLLA fibres, the polymeric matrix can be combined with inorganic materials, such as bioactive glasses (BGs) and glass-ceramics, improving its osteoinductive potential $[11,12]$. Moreover, the PLLA degradation rate can be shaped by several different variables (e.g. chemical structure and crystallinity of the polymer, size and shape of the final material), although, the matching of the rate of degradation and the kinetics of the formation of new bone tissue is still difficult to achieve $[13,14]$. The addition of an inorganic phase allows a better control of the degradation rate of the final PLLA-glass composite, which can be used to manufacture a composite with a suitable degradation rate while progressively being substituted by the new bone that is being formed [15].

BGs are a group of inorganic bioactive materials that are able to form a bone-like hydroxyapatite (HA) layer on their surface capable to strongly bond to hard and soft tissues [11,16-18]. Immediately after the launch of the $45 \mathrm{~S} 5$ Bioglass ${ }^{\circledR}$ in the market (chemical composition by mol: $0.45 \mathrm{SiO}_{2} \cdot 0.06 \mathrm{P}_{2} \mathrm{O}_{5} \cdot 0.245 \mathrm{Na}_{2} \mathrm{O} \cdot 0.245 \mathrm{CaO}$ ) it became the gold standard for bioactive glass materials [16]. Subsequently, a wide range of BGs based on the 4555 composition have been developed and studied for BTE $[19,20]$. The addition of borate glass forming units is one approach that has potential to lower melting temperatures while controlling bio-degradation and increasing conversion rates to HA $[21,22]$. Borosilicate-based BGs (BBGs) have been previously studied as biomaterials and have shown relevant capacity as osteointegrative antibacterial materials to be used in BTE $[23,24]$. BBGs have been also incorporated into scaffolds: Wang et al. [25] successfully incorporated copper doped borosilicate glasses (BBGs-Cu) into a polymeric scaffold for tissue engineering purposes. They observed an improvement in the stability of the glass network and the BBGs-Cu promoted the angiogenesis in rat calvarial defects.

Moreover, in our previous studies we tested a BBG glass composition (e.g. molar ratio: BBG-Sr: $0.05 \mathrm{Na}_{2} \mathrm{O} \cdot 0.35 \mathrm{SrO} \cdot 0.20 \mathrm{~B}_{2} \mathrm{O}_{3}$. $0.40 \mathrm{SiO}_{2}$ ) for their osteogenic capacity. We found that the presence of BBG-Sr glass particles improved the osteogenic differentiation of bone marrow mesenchymal stem cells (BM-MSCs) and induced the formation of mineralised tissue. We also demonstrated that BBG-Sr, at concentrations $\geqslant 18 \mathrm{mg} / \mathrm{mL}$ it was able to eradicate Pseudomonas aeruginosa bacterium [26]. Numerous reports have already associated $\mathrm{Sr}^{2+}$ with bone therapeutic potential [19]. Depending on the concentration, $\mathrm{Sr}^{2+}$ can exerts many effects on bone metabolism at the tissue and cellular levels as well as in bone formation in vivo $[27,28]$. For instance, Marie et al. [27] demonstrated strontium modulates bone cell function (e.g. cell proliferation and differentiation) in vitro stimulating bone formation and inhibiting bone resorption. Moreover, Hesaraki et al. showed that glasses doped with strontium promoted osteoblast proliferation and ALP activity when directly in culture with cells [29]. For instance, $\mathrm{Wu}$ et al. [30] developed a strontium silicate glass which promoted a high ALP activity in BM-MSC cell culture, that was associated with the release of $\mathrm{Sr}^{2+}$ and silica. Also, SantocildesRomero et al. [31] demonstrated that strontium-substituted bioactive glasses promoted osteogenic differentiation of BM-MSCs cultures in the presence of strontium - containing BGs. They showed an increased expression of genes such as Alpl and Bglap. Moreover, Gentleman et al. [32] studied the effect of the release of Si- and Sr-related chemical entities from PCL-SrBG scaffolds, and they detected that the SrBG particles stimulated osteoblast proliferation and ALP activity in relation to the SrBG-free PCL scaffold. Moreover, BM-MSCs are of special interest due to their capacity to differentiation into different lineages (including osteoblastic cells) with appropriate external stimuli and the fact that it is relatively easy to isolate [33]. For that reason, BM-MSCs have received extensive attention as promoters of tissue regeneration.

To our knowledge, the PLLA-BBG-Sr composite membranes fabricated by electrospinning has not been achieved to-date. Therefore, we aimed to evaluate the potential of PLLA-BBG-Sr composite membranes for BTE and bone regeneration. Herein, we investigated the impact of the incorporation of BBG-Sr glass particles in PLLA fibre meshes on their tensile strength and degradation. Moreover, we evaluated the response of BM-MSCs in the presence of PLLA-BBG-Sr fibres, namely in what regards their morphology, proliferation and ability to induce its differentiation.

\section{Experimental}

\subsection{Materials}

All chemicals used for the melt-quenched synthesis were reagent grade: boron oxide $\left(\mathrm{B}_{2} \mathrm{O}_{3}\right.$, Alfa Aesar, Germany), calcium carbonate $\left(\mathrm{CaCO}_{3}\right.$, Sigma-Aldrich, UK), sodium bicarbonate $\left(\mathrm{NaHCO}_{3}\right.$, Sigma-Aldrich, Australia), silica gel $60 \mathrm{M}\left(\mathrm{SiO}_{2}\right.$, Macherey-Nagel, Germany), magnesium oxide (MgO, SigmaAldrich, UK) and strontium carbonate $\left(\mathrm{SrCO}_{3}\right.$, Sigma-Aldrich, Australia). All the chemical reagents used for electrospinning were reagent grade: PLLA with a L-lactide content of $99.6 \%$ and an average $\mathrm{M}_{\mathrm{w}}$ of $69,000 \mathrm{~g} \cdot \mathrm{mol}^{-1}$ (Cargill Dow LLC, USA), dichloromethane (Sigma-Aldrich, UK), and the anionic surfactant docusate sodium salt (Sigma-Aldrich, UK).

The BBGs were produced as described elsewhere [34]. Briefly, the appropriate amounts of $\mathrm{SiO}_{2}, \mathrm{~B}_{2} \mathrm{O}_{3}, \mathrm{NaHCO}_{3}$, and $\mathrm{CaCO}_{3}$ or $\mathrm{MgO}$ or $\mathrm{SrCO}_{3}$, were accurately mixed with ethanol (Sigma, Portugal) in a porcelain pestle and mortar, fully dried overnight and transferred to a platinum crucible (ZGS platinum, Johnson Matthey, UK). Each batch $(\sim 50 \mathrm{~g})$ was heated to $1450{ }^{\circ} \mathrm{C}$ in air for $1 \mathrm{~h}$ and, subsequently, the melt was quickly poured into an ice-water bath at $\sim 0{ }^{\circ} \mathrm{C}$ to form a glass frit. Afterwards, the glasses of general formula $0.05 \mathrm{Na}_{2} \mathrm{O} \cdot x \mathrm{MgO} \cdot y \mathrm{CaO} \cdot(0.35-x-y) \mathrm{SrO} \cdot 0.2 \mathrm{~B}_{2} \mathrm{O}_{3}$. $0.40 \mathrm{SiO}_{2}$ (molar ratio, where $x, y=0.35$ or 0.00 , and $x \neq y$ ) were ground into an Agate mortar (RETSCH, Germany) to obtain microparticles and sieved to a size $<45 \mu \mathrm{m}$ to be homogeneously incorporated in the polymeric matrix during the fabrication of the fibres. The density of the BBGs was measured by a Multi pycnometer (Quantachrome Instruments, USA) under helium at $110^{\circ} \mathrm{C}$ using $\sim 5 \mathrm{~g}$ of each sample.

\subsection{Membranes preparation}

PLLA-BBG-Sr membranes were fabricated by electrospinning inside a fume hood cabinet for safe solvent evaporation and in 
order to prevent that the turbulent air interferes in the formation of the fibres. The PLLA concentration, the ratio of PLLA/ BBG-Sr and the process parameters (e.g. applied voltage, flow rate and distance from the collector) were optimized in order to obtain uniform membrane of fibres. The final electrospun membranes were fabricated using PLLA dissolved in dichloromethane $(16 \% \mathrm{w} / \mathrm{v})$ where the BBGs microparticles were suspended (10\% w/w BBGs/ PLLA). Docusate sodium salt ( $1.2 \% \mathrm{w} / \mathrm{w}$ relative to PLLA) was used to help the homogenization of the solution avoiding the formation of agglomerates. The PLLA and PLLA-BBG-Sr solutions were stirred overnight and sonicated 5 min before use to remove air bubbles. The set up was mounted using a high voltage supplier, a syringe pump (Baxter AS50) with a 20 gauge metal needle (Fisnar, New Jersey, USA) and a conductive collector. The PLLA and PLLA-BBG-Sr solutions were drawn up into a $1 \mathrm{ml}$ syringe (BD Plastipak, New Jersey, USA). The solutions were electrospun using $17 \mathrm{kV}$ and a flow rate of $3 \mathrm{~mL} / \mathrm{h}$ at a $19 \mathrm{~cm}$ distance between the collector and needle. The electrospun membranes were dried in a fume hood, at room temperature, for $24 \mathrm{~h}$, collected and stored in a desiccator at room temperature.

\subsection{Characterisation of electro-spun membranes}

\subsubsection{Scanning electron microscopy (SEM)}

The Scanning Electron Microscopy (Leica Cambridge S360 microscope, equipped with an energy dispersive spectrometer, UK) was used to assess the surface morphology of the fabricated PLLA and PLLA-BBG-Sr membranes. Prior to the analysis, all the scaffolds were sputter-coated with gold. The micrographs were acquired using a beam energy of $5.0 \mathrm{kV}$ and working distance of $\sim 5.2 \mathrm{~mm}$.

\subsubsection{Micro-computed tomography $(\mu \mathrm{CT})$}

Micro-computed tomography ( $\mu-\mathrm{CT})$ was carried out on a highresolution $\mu$-CT scanner (SkyScan1272, Bruker, Kontich, Belgium), using a pixel size of $9.8 \mu \mathrm{m}$ and integration time of $160 \mathrm{~ms}$. The $\mathrm{X}$-ray source was set at $50 \mathrm{kV}$ of energy and $200 \mu \mathrm{A}$ of current. Approximately 400 projections were acquired over a rotation range of $180^{\circ}$ with a rotation step of $0.60^{\circ}$. Data sets were reconstructed using standardised cone-beam reconstruction software (NRecon v1.6.10.2, SkyScan). The output format for each sample was a series of 601 bitmap images $(1224 \times 1224$ pixels). 3D virtual models of representative regions in the bulk of the scaffolds were created applying colour channel thresholds and visualised using an image processing software (CTvox).

\subsubsection{Mechanical tests}

Tensile strength and modulus of the PLLA and PLLA-BBG-Sr membranes were measured using a Uniaxial Universal Testing Machine (Instron 4505, USA) according to the standard ASTM D 638. The membranes were cut into strips of $50 \mathrm{~mm}$ length, $10 \mathrm{~mm}$ width and $0.1 \mathrm{~mm}$ thickness. The tests were conducted using a $1 \mathrm{kN}$ load cell, with a gauge length of $20 \mathrm{~mm}$ and a crosshead speed of $5 \mathrm{~mm} \cdot \mathrm{min}^{-1}$ until rupture. The tensile force was taken from the stress-strain curves as the maximum stress hold by the samples. Tensile modulus was estimated from the initial slope of the stress-strain curve (between $0.5 \%$ and $1 \%$ strain) using the linear regression method. The average and standard deviations were determined using 5 specimens per composition.

\subsubsection{Degradation assay}

The electrospun membranes ( $\mathrm{n}=3$ per time point) were immersed in PBS (Sigma-Aldrich, UK) at a ratio of 10:10 (membrane (mg): PBS $(\mathrm{mL})$ ) for 7, 14, 21, and 28 days in a watershaking bath at $60 \mathrm{rpm}$ and $37^{\circ} \mathrm{C}$. Each immersion solution was filtered and the $\mathrm{pH}$ measured (Crison Instruments, Spain). Inductive coupled plasma (ICP) analysis was performed to determine the concentrations of Si, B and Sr in solution. The absorption was measure at specific wavelengths $(\lambda=251.611 \mathrm{~nm}$ for $\mathrm{Si}, \lambda=249.773 \mathrm{~nm}$ for $\mathrm{B}$ and $\lambda=407.771 \mathrm{~nm}$ for $\mathrm{Sr}$ ) and the concentrations were determined using calibration curves obtained with standard solutions (Alfa Aesar) prior to the analysis of the samples.

The membranes were removed from PBS, the excess surface water was removed and the samples were immediately weighed. Afterwards, the samples were dried in the oven at $37^{\circ} \mathrm{C}$, to constant weight, recording the final mass of the membranes. The water uptake (WU) was calculated according to Eq. (1):

$W U(\%)=\left(m_{t p}-m_{f}\right) / m_{f} \times 100$

where $\mathrm{m}_{\mathrm{tp}}$ is the wet mass of the specimen at the specific time (days), and $\mathrm{m}_{\mathrm{f}}$ is the final mass after immersion and drying. The weight loss (WL) was calculated according to Eq. (2):

$W L(\%)=\left(m_{f}-m_{i}\right) / m_{i} \times 100$

where $\mathrm{m}_{\mathrm{f}}$ is the mass of the dried membranes after its immersion in water, and $\mathrm{m}_{\mathrm{i}}$ is the mass of the dried membranes before immersion in PBS.

Thermogravimetric analysis (TGA; Q500, TA Instruments, USA) was also used to quantify the changes in the weight (mass) of the membranes during the degradation process. In addition, thermal analysis was performed to determine the amount of BBG-Sr (non-combustible) glass particles that was compounded with PLLA to produce the composite membranes. Experiments were performed in platinum pans, at a heating rate of $40 \mathrm{~K} \cdot \mathrm{min}^{-1}$ from 50 to $700{ }^{\circ} \mathrm{C}$ in an oxygen atmosphere.

\subsection{In vitro culture of BM-MSCs on electrospun membranes}

\subsubsection{Isolation and expansion of BM-MSCS}

Bone marrow mesenchymal stem cells (BM-MSCs) were isolated from bone marrow of 4-5 week-old male Wistar rats according to the method established by Maniatopoulos et al. [35] and recently used by Santocildes-Romero [31]. BM-MSCs were expanded in basal medium consisting of Dulbecco's modified Eagle's medium (DMEM; Sigma-Aldrich, UK), supplemented with $100 \mathrm{U} / \mathrm{mL}$ penicillin (Sigma-Aldrich, UK) and $100 \mu \mathrm{g} / \mathrm{mL}$ streptomycin (Sigma-Aldrich, UK). Cells were cultured at $37^{\circ} \mathrm{C}$ in an atmosphere of $5 \% \mathrm{CO}_{2}$.

\subsubsection{Culture of BM-MSCS}

Prior to the in vitro studies, BM-MSCs, at passage 2, were harvested and seeded at a density of $3 \times 10^{4}$ cells per membrane of $\varnothing=6.5 \mathrm{~mm}$ held in plastic inserts (CellCrown ${ }^{\mathrm{TM}} 24$, Scaffdex, UK). Cells were cultured for 7, 14 and 21 days under static conditions. All scaffold conditions were cultured in basal and osteogenic differentiation media (basal medium supplemented with $50 \mu \mathrm{g} / \mathrm{mL}$ L-ascorbic acid, $10 \mathrm{mM} \beta$-glycerophosphate and $10^{-8} \mathrm{M}$ dexamethasone).

\subsubsection{BM-MSCs proliferation, viability and morphology}

2.4.3.1. Morphological evaluation of cultured cells. After each timepoint the cells cultured on the membranes were washed with PBS and fixed with $4 \%$ formalin solution $(0.5 \mathrm{~mL})$ for $15 \mathrm{~min}$ at room temperature (RT). The cells were then washed with PBS, containing $0.2 \%$ Triton $\mathrm{X}$, for $2 \mathrm{~min}$. After the fixation and permeation steps, the cells were washed again with PBS and stained with 4,6diamidino-2-phenyindole dilactate (1:1000 DAPI, Sigma-Aldrich, UK) for 2 min at RT, and phalloidin-tetramethylrhodamine B isothiocyanate (Sigma-Aldrich, UK) for $1 \mathrm{~h}$ at RT. Finally, the cells were washed and observed using an Axioplan 2 imaging fluorescent microscope with a digital camera QIC AM 12-bit (Zeiss, UK). 
2.4.3.2. Cell viability and proliferation (PrestoBlue ${ }^{\circledR}$ and PicoGreen ${ }^{\circledR}$ assays). The PrestoBlue ${ }^{\circledR}$ reagent (Fisher Scientific, UK) is a resazurin-based solution that is reduced to resorufin by viable cells which can be detected fluorometrically. The cell viability assay was executed according to the manufacturer's instructions. In brief, the PrestoBlue $^{\circledR}$ reagent was added to a final concentration of $10 \%$ to the cell culture medium and the cultures incubated for $1 \mathrm{~h}$ at $37^{\circ} \mathrm{C}$. $200 \mu \mathrm{L}$ samples of the culture medium were removed and placed in 96-well plates and the resorufin fluorescence quantified spectrophotometrically using a plate reader (Tecan Infinite M200). The fluorescence was determined at an excitation wavelength of $560 \mathrm{~nm}$ and emission wavelength of $590 \mathrm{~nm}$. The metabolic activity was presented in fluorescence values and compared with the control (cell cultured onto PLLA membranes under basal medium conditions).

The PicoGreen ${ }^{\circledR}$ dsDNA reagent (Invitrogen, USA) is an ultrasensitive fluorescent nucleic acid dye for quantification of double-stranded DNA (dsDNA) in solution. This assay enables measurement of cell proliferation. After each culturing period, the cells were washed with PBS and incubated at $37^{\circ} \mathrm{C}$ for $3 \mathrm{~h}$ followed by a freezing step at $-80^{\circ} \mathrm{C}$ for overnight in ultra-pure water $(1 \mathrm{~mL})$ to ensure the cell lysis. Finally, the fluorescence was determined at an excitation wavelength of $485 \mathrm{~nm}$ and emission wavelength of $528 \mathrm{~nm}$. The DNA concentration was presented in $\mu \mathrm{g} / \mathrm{mL}$ and compared with the control (cell cultured onto PLLA membranes under basal medium conditions).

\subsubsection{Alkaline phosphatase quantification}

The concentration of alkaline phosphatase (ALP) was determined for all the cell culture time points, using the lysates used for the DNA quantification and the Alkaline Phosphatase, Diethanolamine Detection kit (Sigma-Aldrich, UK), which is based on the conversion of p-nitrophenyl phosphate (pNPP) to free p-nitrophenol by ALP. In brief, a buffered pNPP solution was prepared and equilibrated at $37^{\circ} \mathrm{C}$. Afterwards, $2 \%(\mathrm{v} / \mathrm{v})$ of sample or control lysate was added. Immediately after mixing the absorbance was read at $405 \mathrm{~nm}$ in a plate reader (Tecan Infinite M200) for $\approx 5$ min. An ALP standard solution was used as control and buffer as blank. The units were calculated according to the following Eq. (3):

$\frac{\left(\Delta A_{405 \mathrm{~nm}} / \min \text { Test }-\Delta A_{405 \mathrm{~nm}} / \min \text { Blank }\right) \times d f \times V_{F}}{185 \times V_{E}}$

where $\mathrm{df}=$ dilution factor; $\mathrm{V}_{\mathrm{F}}=$ Volume of final solution; $18.5=$ millimolar extinction coefficient of pNPP at $405 \mathrm{~nm}$ and $\mathrm{V}_{\mathrm{E}}=$ Volume of samples/ALP standard solution.

\subsubsection{RNA isolation and real-time quantitative polymerase chain reaction ( $r$ PCCR)}

RNA was extracted with Tri-reagent (Sigma-Aldrich, UK) according to the manufacturer's instructions. The RNA concentration was determined by microspectrophotometry (NanoDrop 1000). The cDNA synthesis was performed using the qScript cDNA synthesis kit (Quanta BioSciences, VWR) with 100 ng of RNA template in a final volume of $20 \mu \mathrm{L}$. Cycling was as follows: 1 cycle at $22{ }^{\circ} \mathrm{C}, 5 \mathrm{~min} ; 1$ cycle at $42{ }^{\circ} \mathrm{C}, 30 \mathrm{~min} ; 1$ cycle at $85^{\circ} \mathrm{C}, 5 \mathrm{~min}$. The amplification of the target cDNA was performed using the PerfeCTa SYBR Green FastMix (Quanta BioSciences) with $1 \mu \mathrm{L}$ of cDNA, 200 nM of each primer (Table 1 ) in a final volume of $20 \mu \mathrm{L}$. Real time-PCR cycling was as follows: 1 cycle at $95^{\circ} \mathrm{C}, 2 \mathrm{~min} ; 44$ cycles at $95{ }^{\circ} \mathrm{C} ; 10 \mathrm{~s}$ at gene annealing temperature (Table 1 ); $30 \mathrm{~s}$ at $72^{\circ} \mathrm{C}$; followed by dissociation curve analysis. All the reactions were carried out on a PCR cycler Mastercycler Realplex (Hamburg, Germany). The transcripts expression data were normalised to the housekeeping gene glyceraldehyde-3-phosphate dehydrogenase (Gapdh) in each sample. The quantification was performed
Table 1

Primers used for qPCR.

\begin{tabular}{llc}
\hline Gene & $\begin{array}{l}\text { Primer sequence } 5^{\prime}-3^{\prime} \\
\text { Forward/ reverse }\end{array}$ & $\operatorname{Tm}\left({ }^{\circ} \mathrm{C}\right)$ \\
\hline Osteocalcin (Bglap) & $\begin{array}{l}\text { CATCTATGGCACCACCGTTT } \\
\text { AGAGAGAGGGAACAGGGAGG }\end{array}$ & 60.0 \\
Osteopontin (Spp1) & $\begin{array}{l}\text { ATCTCACCATTCCGATGAATCT } \\
\text { CAGTCCATAAGCCAAGCTATCA }\end{array}$ & 60.0 \\
Osterix (Sp7) & $\begin{array}{l}\text { CACTGGCTCCTGGTTCTCTC } \\
\text { CCACTCCTCCTCTTCGTGAG }\end{array}$ & 60.0 \\
Alkaline phosphatase (Alpl) & $\begin{array}{l}\text { TGCCTTACCAACTCATTTGTG } \\
\text { ACGCGATGCAACACCACTC }\end{array}$ & 57.4 \\
\hline
\end{tabular}

according to the Livak method ( $2^{-\Delta \Delta \mathrm{Ct}}$ method [36]), considering as calibrator at each time point the PLLA - basal medium for PLLA - osteogenic medium and PLLA-BBG-Sr basal medium for PLLA-BBG-Sr osteogenic medium (threshold $=1$ ).

\section{Results and discussion}

\subsection{Characterisation of electrospun membranes}

The PLLA and PLLA-BBG-Sr (10\% w/w BBG-Sr/PLLA) composite membranes were successfully obtained by electrospinning. TGA analysis confirmed that we successfully incorporated $10 \%(\mathrm{w} / \mathrm{w})$ of BBG-Sr microparticles in the PLLA fibres. The morphology, microstructure and fibre integrity of the electrospun PLLA and PLLA-BBG-Sr membranes was characterised by SEM and $\mu$ CT. Electrospun PLLA and PLLA-BBG-Sr membranes are composed of smooth and uniform fibres. No large particles were detected (Fig. 1a and c), however, the formation of small agglomerates in the membranes containing the $\mathrm{BBG}-\mathrm{Sr}$ microparticles was observed [37]. Fig. 1c highlights in red circles the BBG-Sr microparticles incorporated into the fibres. The homogeneous distribution of the BBG-Sr microparticles in the PLLA-BBG-Sr membrane could be observed in the $2 \mathrm{D}$ virtual model representatively obtained using an image processing software CTvox of $\mu \mathrm{CT}$ images (Fig. 1d), in which the red colour represents the BBG-Sr microparticles distributed into the composite membranes in green. As expected, the PLLA membranes do not present any particles in their structure (Fig. 1b).

The mechanical properties of the biomaterials are important in providing physical support for cell growth and migration matching those of the tissue at the site of implantation [38]. Fig. 2a presents the stress-strain curves obtained for the PLLA and PLLA-BBG-Sr membranes under tensile load up to a strain of $50 \%$. The PLLA-BBG-Sr membranes showed the highest tensile strength $(0.75 \pm 0.7 \mathrm{MPa})$, when compared to the PLLA membranes $(0.55 \pm 0.6 \mathrm{MPa})$, indicating that the incorporation of BBG-Sr reinforced the membranes. The same trend was observed for the Young's modulus (Fig. 2b), calculated from the initial linear slope of the stress-strain curves, where the loading of BBG-Sr microparticles improved the modulus from $14.6 \pm 3.8 \mathrm{MPa}$ (PLLA) to $24.7 \pm 5.3 \mathrm{MPa}$ (PLLA-BBG-Sr). This might be due to an increase in rigidity through the filler effect, in which the microparticles encumber the movement of the polymer chains and the amount of extendable material in the membrane. On the other hand, while the incorporation of the microparticles in PLLA-BBG-Sr membranes can affect the tensile strength, where there is no interaction between fibres and particles. The availability of cations derived from the incorporated microparticles may contribute to the crosslinking of the carboxylic acid groups (that are present in the chain ends of the PLLA) through the formation of carboxylates. These carboxylate cross-links induce the interlocking of the 


\section{PLLA}
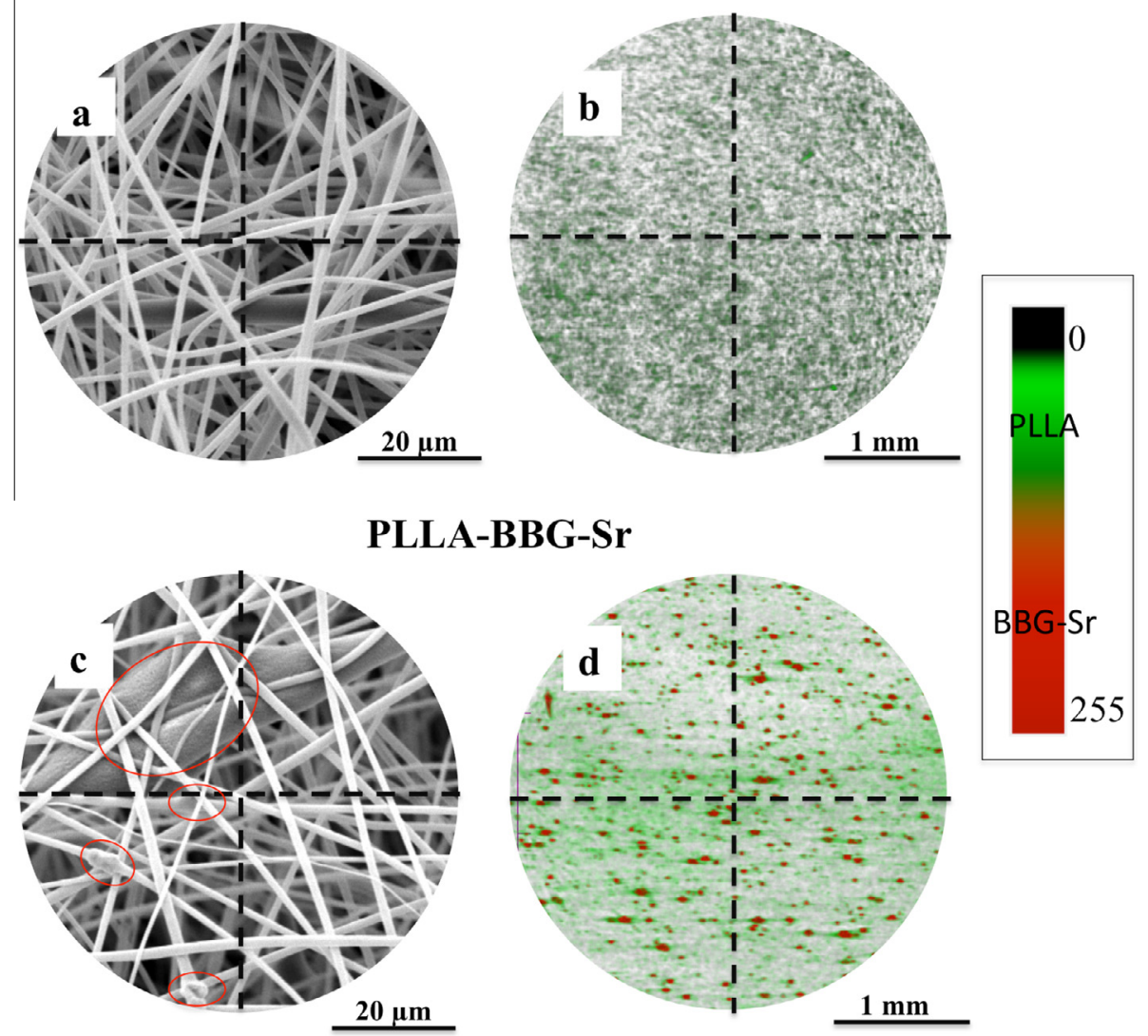

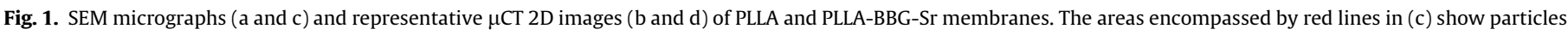

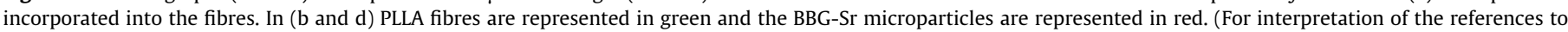
colour in this figure legend, the reader is referred to the web version of this article.)
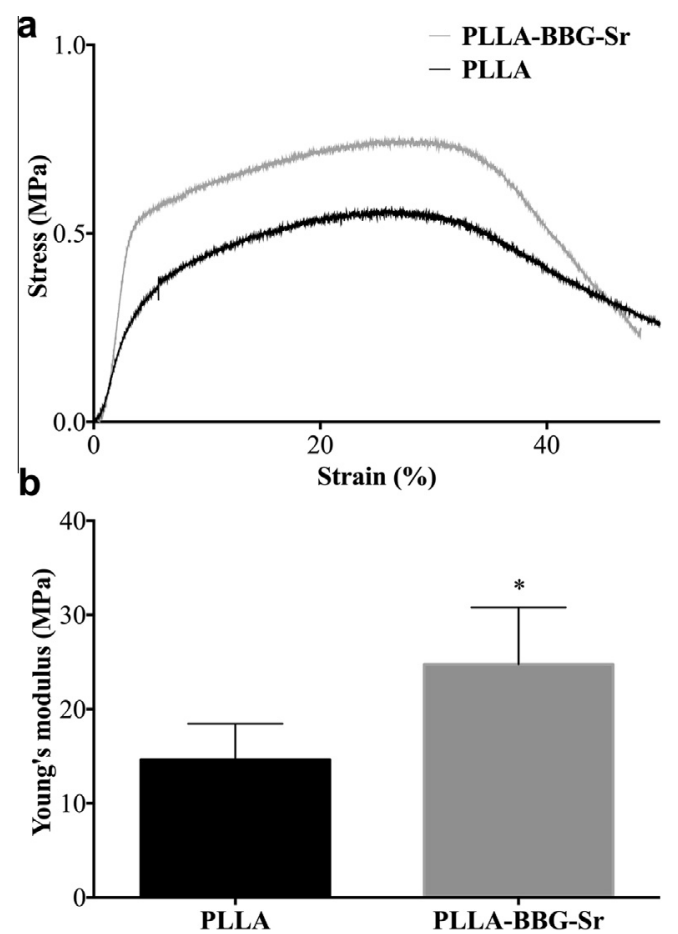

Fig. 2. (a) Stress-strain curves of PLLA and PLLA-BBG-Sr membranes and their respective (b) Young's modulus determined from the initial slope of the curves. The data was analysed by non-parametric statistics; data analysis using the MannWhitney test revealed a significance of $\mathrm{p}<0.05\left(^{*}\right)$. membranes, resulting in an improvement of the Young modulus [39]. As demonstrated by Thomas et al. [40], small concentrations of nano hydroxyapatite (HA) resulted in a reinforcement of PCL. It was observed by Jeong et al. [41] in an analogous way, that the addition of increasing concentrations of HA increasingly enhanced the mechanical properties of the composite, namely their tensile strength and Young's modulus. Therefore, our results support that, with the inclusion of BBG-Sr microparticles into the PLLA matrix, it is possible to tune their mechanical properties, contributing to be a closer match of those from the living tissue at the site of implantation.

\subsection{Degradation of the electrospun membranes}

A biomaterial suitable for promoting bone regeneration in a tissue engineering perspective should present an appropriate biodegradability. The BTE concept is based on the substitution of the biomaterial by new bone. This requires that the degradation rate of the biomaterial matches that of new bone formation, in order to occur a progressive substitution of the biomaterial by the new bone tissue [15,42]. The degradation profiles of PLLA and PLLA-BBG-Sr membranes (Fig. 3) were evaluated from the weight loss and water uptake, as well as the release of chemical species from the membranes and $\mathrm{pH}$ of the immersion solutions. The water uptake and weight loss data showed differences between the PLLA and the PLLA-BBG-Sr membranes (Fig. $3 \mathrm{~b}$ and $\mathrm{d}$ ). Both membranes presented steady and continuous water uptake and weight loss from week 2 to week 3 . However, the PLLA-BBG-Sr membranes presented a more pronounced 'burst' of 

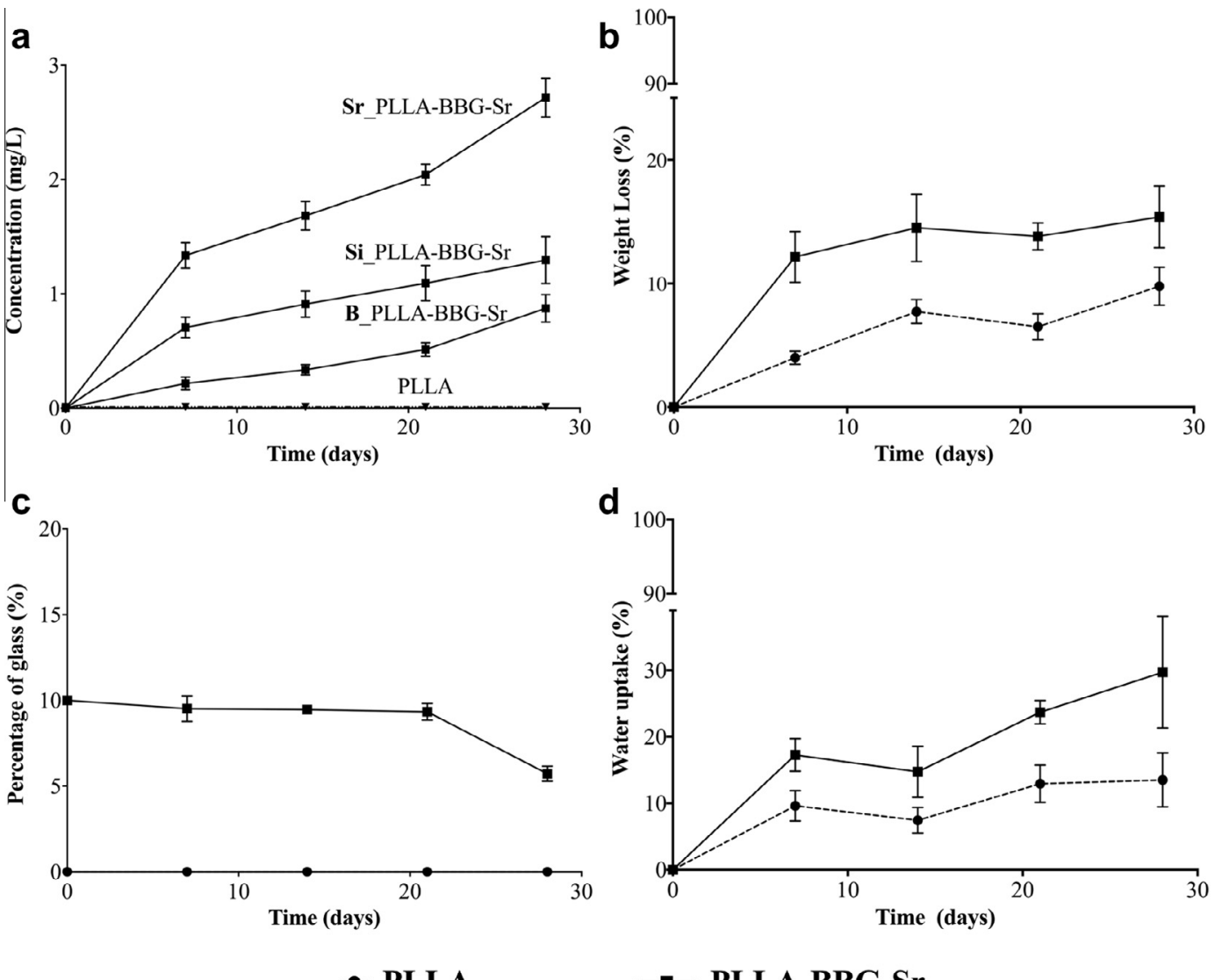

$\rightarrow$ PLLA

$\rightarrow$ PLLA-BBG-Sr

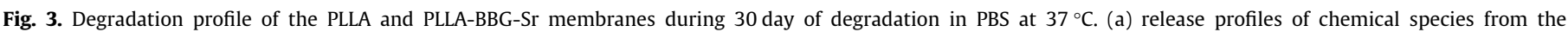
PLLA-BBG-Sr membranes; (b and d) WU and WL of the PLLA and PLLA-BBG-Sr membranes; and (c) percentage of glass (w/w) in the studied membranes.

weight loss during the first week. The differences between the two membranes during this initial stage of degradation might be attributed to the penetration of the PBS solution through the polymer/glass interface and/or surface cracks that facilitated the degradation of the material and enhanced the water uptake. Another possibility is related with a superficial hydrolytic degradation generated by the leaching of chemical species from the glass particles $[43,44]$. The $\mathrm{pH}$ measurements presented no significant variation of $\mathrm{pH}$ for both PLLA and PLLA-BBG-Sr membranes over time. An initial burst release of B, Si and $\mathrm{Sr}$ from the PLLA-BBG-Sr membranes into the immersion solution was observed (Fig. 3a). The release of such chemical species can only have originated from the BBG-Sr microparticles, corroborating the possibility of the origin of the weight loss being mainly derived from the hydrolytic degradation of the glass particles. During the last week of degradation (21-28 days) it was also noticed that an increase in the PLLA-BBG-Sr weight loss that correlates with the reduction of glass percentage determined by TGA (Fig. 3c) and the increased concentration of mainly $\mathrm{B}$ and $\mathrm{Sr}$-containing chemical species, that were leached from the glass particles to the immersion medium (detected by ICP). The increase on the degradation is clear during the last week; however, the leaching observed by ICP during the first 3 weeks is not corroborated by the TGA analysis. In this case, the presence of phosphates from the solution buffer might generate a surface layer of strontium phosphate that limits the mass exchange from the glass particles to the immersion solution. In this case, the weight loss derived from the leaching of B, Si and $\mathrm{Sr}$ from the glass particles is partially compensated by the deposition of phosphates. The fact that the PLLA-BBG-Sr composite membranes lost twice the mass during the degradation process suggests that the incorporation of BBG-Sr into PLLA fibres increased the degradability and the degradation rate of the membranes, as well as the release of active chemical species to the surrounding medium [15].

\subsection{In vitro biological evaluation}

\subsubsection{Morphology, viability and proliferation of the BM-MSCS}

Apart from the suitable chemical and physical properties of the biomaterial, it is also crucial to evaluate if they are cytotoxic. To this purpose, direct contact assays are widely used as a preliminary screening for biomaterials [45]. Fluorescence microscopy images (Fig. 4a) show the morphology of BM-MSCs cultured in the presence of PLLA and PLLA-BBG-Sr membranes in basal or osteogenic medium (Fig. 4a, Basal and Osteo, respectively). The attached BM-MSCs displayed a well-spread morphology and several cellto-cell contacts. As expected, the PrestoBlue ${ }^{\circledR}$ and PicoGreen ${ }^{\circledR}$ data (Fig. $4 \mathrm{~b}$ and c) demonstrated that the cells proliferated over the time course under basal conditions, which is consistent with the morphology images (Fig. 4a). There is also and increase on their metabolic activity (day 7 versus day 21). However, under osteogenic conditions BM-MSCs did not proliferate and exhibited an increase in metabolic activity (day 7 versus day 21 ), which suggested the occurrence of differentiation $[8,46]$. PLLA and PLLABBG-Sr electrospun membranes were found to be a non-cytotoxic support for BM-MSCs attachment and proliferation (Fig. 4) without any adverse effect caused by the addition of BBG-Sr particles. In addition, the randomly distributed fibres and porous structure of electrospun membranes should enable an efficient transfer of nutrients, as well as support suitable cell penetration into the bulk of the membranes. 
a Day 7 Day 14 Day 21

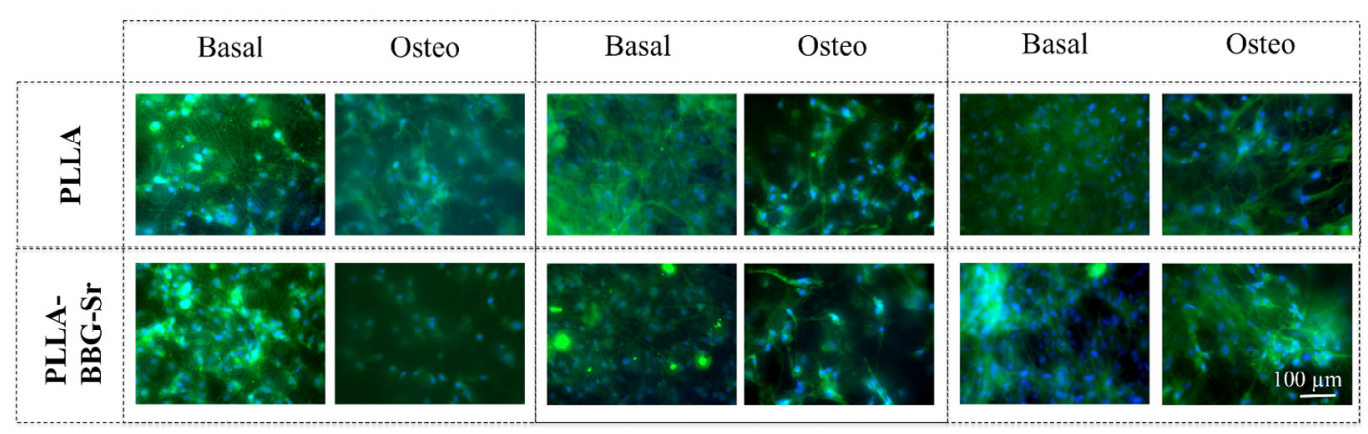

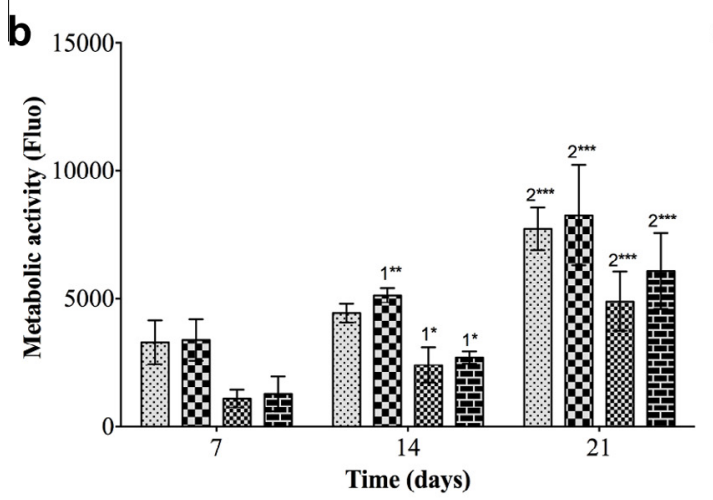

PLLA_basal

Q PLLA-BBG-Sr_basal

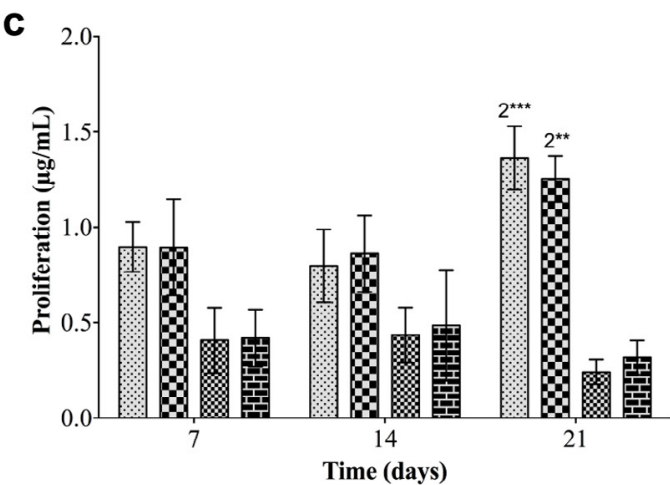

宔 PLLA-BBG-Sr_osteo

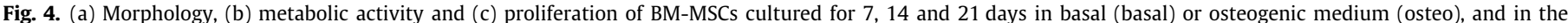

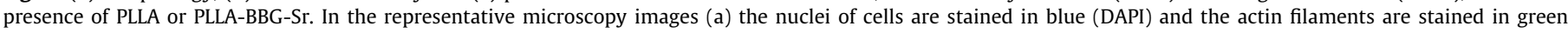

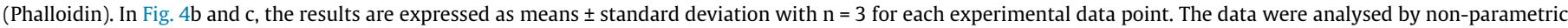

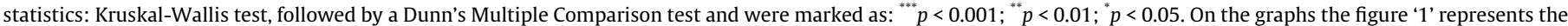

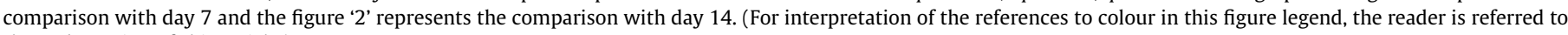
the web version of this article.)

\subsection{Osteogenic differentiation markers}

It has been demonstrated that the release of chemical species containing Si, B or Sr from BBG-Sr glass particles could stimulate the osteoblast proliferation and differentiation. The ALP activity is commonly related with functional activity of the bone-derived cells, such as their osteogenic differentiation and the onset of mineralisation $[37,47]$. In order to execute a preliminary evaluation on the capacity of the PLLA-BBG-Sr membranes to induce osteogenic differentiation, we cultured BM-MSCs onto them and quantified the ALP activity after 7, 14 and 21 days (Fig. 5b). SEM was also used to check for mineral deposits and the mineralisation stage of the BM-MSCs (Fig. 5a). In the SEM micrographs it is possible to confirm that the BM-MSCs create a cell layer attached to the PLLA and PLLA-BBG-Sr membranes. However, in the case of BM-MSCs cultured under osteogenic conditions, formation of phosphate deposits at the later time points (Fig. 5a, day 21) were observed. In contrast, ALP activity (Fig. 5b) increased during the time of culture under osteogenic conditions, suggesting that the addition of BBG-Sr microparticles increased the ALP expression levels. Therefore, the PLLA-BBG-Sr membranes stimulated the BM-MSCs to initiate their osteogenic differentiation. This stimulus is very likely to be related with the presence of the $\mathrm{B}, \mathrm{Si}$ and $\mathrm{Sr}$ chemical species leaching from the BBG-Sr glass particles to the surrounding medium $[48,49]$, as also shown in the ICP data (Fig. 3a). Comparatively, some authors [31,32] studied the effect of the release of $\mathrm{Si}$ and $\mathrm{Sr}$ chemical species from BG, and found that they stimulate cell differentiation. Other authors have also demonstrated improvements in cell differentiation and bone mineralisation by the addition of glass particles into polymeric matrices $[6,37,50]$. As an example, Santocildes-Romero [9], fabricated electrospun membranes incorporating $10 \%$ BGSr glass particles with increasing $\mathrm{Sr}$ content with no cytotoxic effects on osteosarcoma cells; Rajzer and co-workers [37] verified that the addition of $20 \%$ hydroxyapatite ceramic particles into PLDL electrospun composites could direct HAp mineralisation in cell culture; Ren et al. [6] also showed that PCL-SrBG electrospun scaffolds enhanced the ALP activity of MC3T3-E1 cells in the presence of osteogenic media in comparison with the glass-free PCL scaffolds after 21 days of culture. Hence, the addition of BBG-Sr glass particles to PLLA fibres accelerated the BM-MSCs osteogenic differentiation (ALP activity, Fig. 5a), in the presence of osteogenic media after 14 days of cell culture, showing an higher increase after 21 days of cell culture.

Complementary to the reported viability, proliferation and ALP activity data, the differentiation level of BM-MSCs cultured onto PLLA and PLLA-BBG-Sr membranes was assessed by quantitative PCR of selected bone-specific gene transcripts. In the literature it is well described that osteogenic differentiation of BM-MSCs has three phases: the proliferative phase, which is followed by the ECM synthesis and maturation, and lastly the mineralisation phase $[51,52]$. Therefore, in this study the osteogenic potential of the cells was evaluated at day 14 and 21 using the expression pattern of the representative osteogenic markers: Alpl, Spp1, Bglap and Sp7 [8,53]. The transcripts expression data were normalized against the housekeeping gene Gapdh and the quantification performed according to the Livak method ( $2^{-\Delta \Delta \mathrm{Ct}}$ method), considering the 


\section{a}

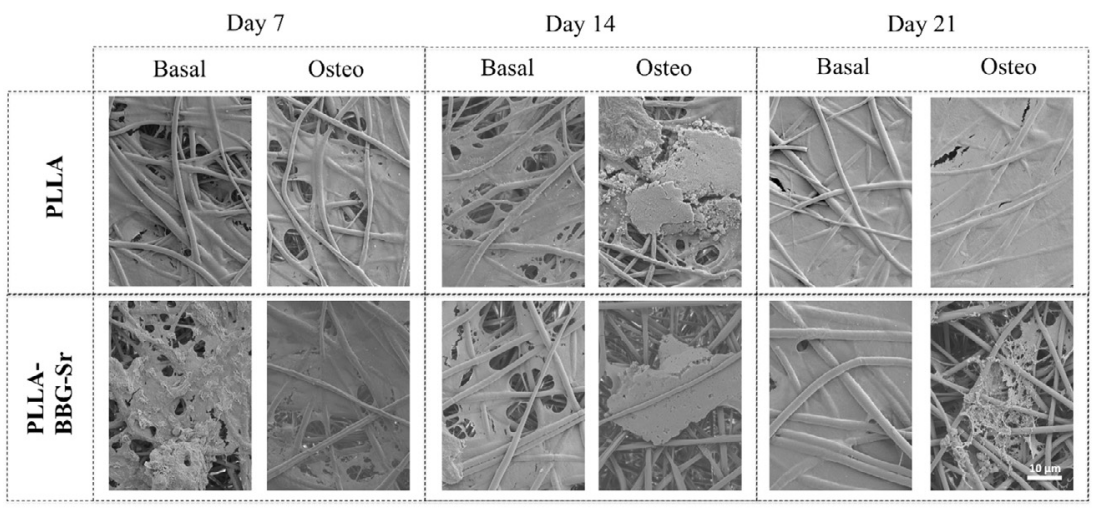

b

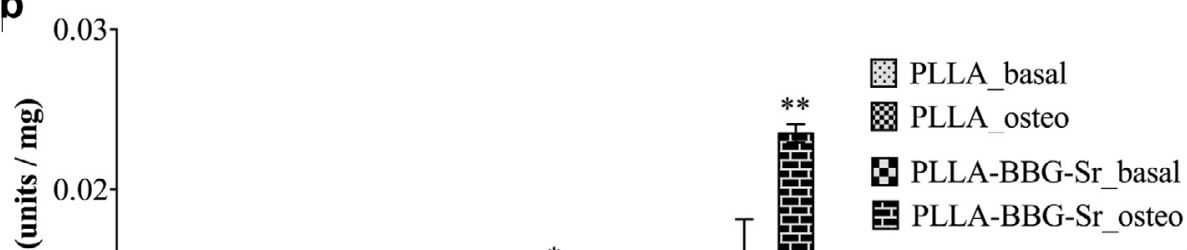

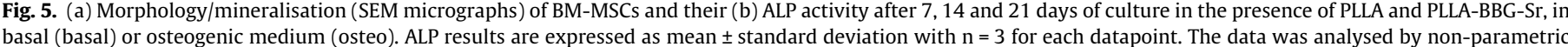

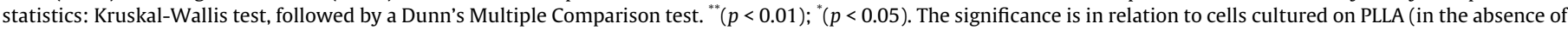
BBG-Sr glass particles) under the same culture conditions.

PLLA (basal medium) as calibrator for PLLA (osteogenic medium) and PLLA-BBG-Sr (basal medium) as calibrator for PLLA-BBG-Sr (osteogenic medium). Commonly, Alpl acts as early marker of the osteoblastic phenotype. This gene expression level is known to increase with the progression of osteoblastic differentiation [54]. According to the gene expression data (Fig. 6a) there is a significant up-regulation of Alpl in the BM-MSCs cultured onto PLLA-BBG-Sr membranes at 14 and 21 days of culture in relation to BM-MSCs cultured onto PLLA membranes (all under osteogenic medium). Again, Ren et al. found the same Alpl gene up regulated in MC3T3 cells cultured in PCL-SrBG membranes, but only after 21 days of culture. These observations are consistent with the quantification of the ALP activity (Fig. 5b), in which there was a significantly higher activity for the BM-MSCs cultured onto PLLA-BBG-Sr (under osteogenic medium) after 14 days of culture, when compared with the cells cultured onto PLLA $[48,49]$. Regarding the maturation and mineralisation phase, we investigated the expression of the genes that encode two non-collagenous proteins present with the ECM of bone, e.g. Spp1 and Bglap. Spp1 is a protein found in bone, teeth, kidneys and epithelial lining tissue; consequently, Spp1 cannot be considered bone-specific, although it is associated to bone related functions $[8,46]$. The Spp1 gene expression results (Fig. 6b) showed that at 14 days of culture, BM-MSCs cultured on PLLA presents a high Spp1 expression, which might be related with their important functions in cell adhesion, migration and survival [54]. The fact that Spp1 expression is significantly higher when cultured on PLLA membranes might be a result of proliferation and cell spreading processes. On the other hand, Bglap, which is the second most abundant protein present in bone, is exclusively secreted by osteoblastic cells at the last stage of maturation [46,54]. When cultured on PLLA-BBG-Sr membranes, Bglap expression (Fig. 6c) is significantly higher in relation to the basal medium for 14 and 21 days of culture. This suggests that the combined effect of the osteogenic factors and the dissolution products might be enhancing Bglap gene expression, which may be an indicator of osteoblastic differentiation. This data is in accordance with the report of Strobel et al. [55] showing that BM-MSCs, after 14 days of exposure to cell culture medium containing nanoparticles of Sr-substituted Bioglass, increased their Bglap expression. Finally, $S p 7$ is a wellcharacterised osteoblast specific gene and it is associated with the regulation of numerous other genes (e.g. osteocalcin, osteopontin, bone sialoprotein), and is acknowledged as a late bone marker that plays a key role in the differentiation of preosteoblasts into fully functioning osteoblasts [8,54]. Our data at day 21 registered an increase of $\mathrm{Sp7}$ gene expression in the BM-MSCs cultured on PLLA-BBG-Sr membranes (under osteogenic medium) in relation to the same cells cultured on the same membranes but under basal medium, as well as the cells cultured onto PLLA (under osteogenic and basal medium). These observations are consistent with the overexpression of the other monitored gene Alpl and their protein expression (ALP) for PLLA-BBG-Sr membrane [56]. In fact, Isaac et al. [57] demonstrated that the addition of strontium to bioactive glass particles resulted in a significant up-regulation of Runx2 and Osterix of osteoblastic cells. In summary, the detected overexpression of Alpl, Bglap and Sp7 genes in cells cultured onto PLLA-BBG-Sr membranes show that the incorporation of BBG-Sr glass particles into the PLLA membranes promotes the osteogenic differentiation of BM-MSCs [48]. 
a

Relative expression of Alkaline phosphatase ( $A l p l)$

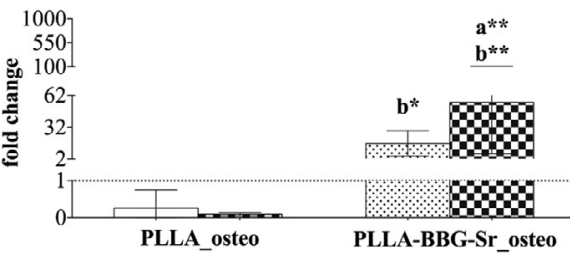

C

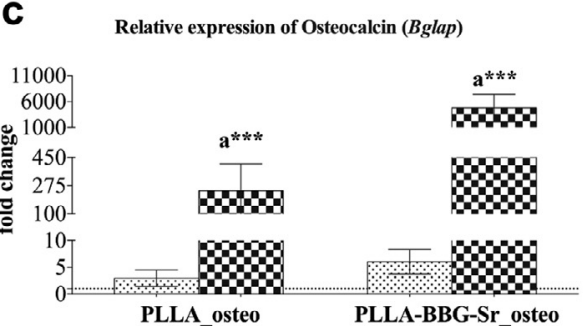

b

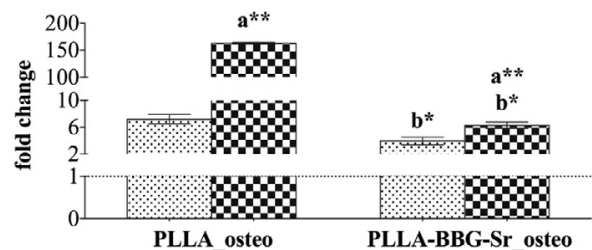

d

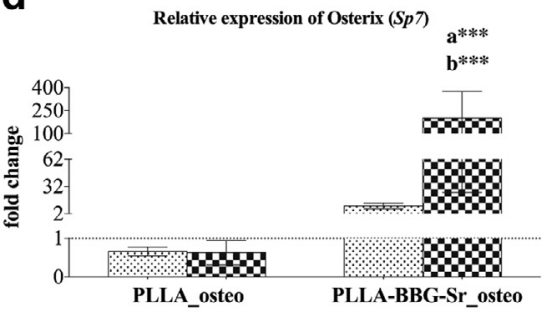

D21 days

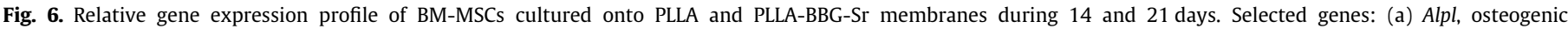

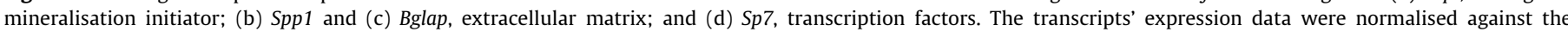

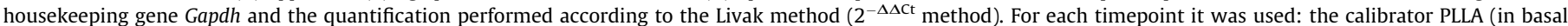

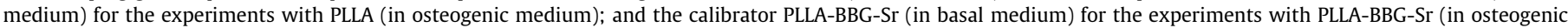

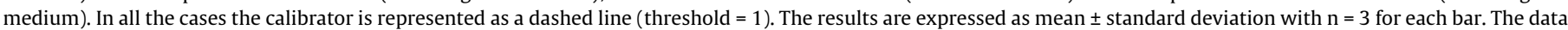

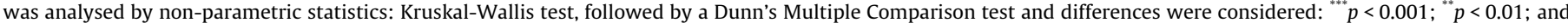

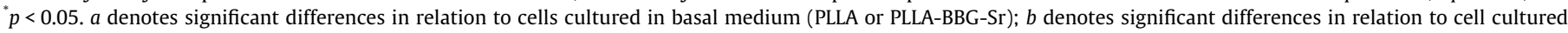
onto PLLA in osteogenic medium.

\section{Conclusion}

Bioactive membranes of PLLA-BBG-Sr composites were successfully prepared using electrospinning. The $\mu \mathrm{CT}$ data evidenced that BBG-Sr microparticles were homogeneously incorporated into the fibres and the membrane structure. Our data indicated that, the addition of BBG-Sr into the PLLA matrix improved the in vitro water uptake and degradability of the membranes. Moreover, the incorporation of the BBG-Sr microparticles improved the mechanical properties of the membranes, namely in their Young modulus and tensile strength. The in vitro biological evaluation confirmed that both PLLA and PLLA-BBG-Sr membranes are able to promote the attachment of BM-MSCs, without any cytotoxic effects. Furthermore, the addition of BBG-Sr microparticles to the PLLA membranes increased the ALP activity (under osteogenic conditions), as well as the BM-MSCs osteogenic differentiation as shown by up-regulation of Alpl, Sp7 and Bglap gene expression in vitro. The present work suggests that the composite of PLLA membranes and BBG-Sr microparticles can be used as a strategy to prepare bioactive composite membranes for bone regeneration.

\section{Acknowledgements}

The authors gratefully acknowledge financial support from Portuguese Foundation for Science and Technology (Ph.D. grant BD/73162/2010), the European Union's Seventh Framework Programme (FP7/2007-2013) under Grant No. REGPOT-CT201231633-POLARIS. This work was also supported by the European Research Council grant agreement ERC-2012-ADG-20120216321266 for the project ComplexiTE and UK EPSRC Centre for Innovative Manufacturing of Medical Devices-MeDe Innovation (EPSRC grant EP/K029592/1).

\section{References}

[1] S. Zhao, M. Zhu, J. Zhang, Y. Zhang, Z. Liu, Y. Zhu, Zhang, Changqing, Three dimensionally printed mesoporous bioactive glass and poly(3hydroxybutyrate-co-3-hydroxyhexanoate) composite scaffolds for bone regeneration, J. Mater. Chem. B 2 (2014) 6106-6118.

[2] T. Jiang, E.J. Carbone, K.W.H. Lo, C.T. Laurencin, Electrospinning of polymer nanofibers for tissue regeneration, Prog. Polym. Sci. 46 (2015) 1-24.

[3] P. Gentile, V. Chiono, I. Carmagnola, P.V. Hatton, An overview of poly(lactic-coglycolic) acid (PLGA)-based biomaterials for bone tissue engineering, Int. J. Mol. Sci. 15 (2014) 3640-3659.

[4] G. Sui, X. Yang, F. Mei, X. Hu, G. Chen, X. Deng, Ryu, Seungkon, Poly-L-lactic acid/hydroxyapatite hybrid membrane for bone tissue regeneration, J. Biomed. Mater. Res. Part A 82A (2007) 445-454.

[5] K. Madhavan Nampoothiri, N.R. Nair, R.P. John, An overview of the recent developments in polylactide (PLA) research, Bioresour. Technol. 101 (2010) 8493-8501.

[6] J. Ren, K.A. Blackwood, A. Doustgani, P.P. Poh, R. Steck, M.M. Stevens, Maria A Woodruff, Melt-electrospun polycaprolactone-strontium substituted bioactive glass scaffolds for bone regeneration. J. Biomed. Mater. Res. Part A 102 (2013) 3140-3153.

[7] B. Gupta, N. Revagade, J. Hilborn, Poly(lactic acid) fiber: an overview, Prog. Polym. Sci. 32 (2007) 455-482.

[8] A. Martins, A.R.C. Duarte, S. Faria, A.P. Marques, R.L. Reis, N.M. Neves, Osteogenic induction of hBMSCs by electrospun scaffolds with dexamethasone release functionality, Biomaterials 31 (2010) 5875-5885.

[9] M.E. Santocildes-Romero, R.L. Goodchild, P.V. Hatton, A. Crawford, I.M. Reaney, C.A. Miller, Preparation of composite electrospun membranes containing strontium-substituted bioactive glasses for bone tissue regeneration, Macromol. Mater. Eng. (2016).

[10] S. Haimi, N. Suuriniemi, A.-M. Haaparanta, V. Ellä, B. Lindroos, H. Huhtala, et al., Growth and osteogenic differentiation of adipose stem cells on PLA/bioactive glass and PLA/ $\beta$-TCP scaffolds, Tissue Eng. Part A 15 (2008) 1473-1480.

[11] S. Bhakta, P. Faira, L. Salata, Neto.P. de Oliveira, C. Miller, R. van Noort Determination of relative in vivo osteoconductivity of modified potassium fluorrichterite glass-ceramics compared with 4555 bioglass, J. Mater. Sci. Mater. Med. 23 (2012) 2521-2529.

[12] L. Hench, The story of bioglass, J. Mater. Sci. Mater. Med. 17 (2006) $967-$ 978.

[13] J.P. Mofokeng, A.S. Luyt, Morphology and thermal degradation studies of melt-mixed poly(lactic acid) (PLA)/poly( $\varepsilon$-caprolactone) (PCL) biodegradable 
polymer blend nanocomposites with $\mathrm{TiO}_{2}$ as filler, Polym. Test. 45 (2015) 93-100.

[14] F. Carrasco, P. Pagès, J. Gámez-Pérez, O.O. Santana, M.L. Maspoch, Processing of poly(lactic acid): characterization of chemical structure, thermal stability and mechanical properties, Polym. Degrad. Stab. 95 (2010) 116-125.

[15] Y. Niu, L. Guo, J. Liu, H. Shen, J. Su, X. An, Bioactive and degradable scaffolds of the mesoporous bioglass and poly(L-lactide) composite for bone tissue regeneration, J. Mater. Chem. B 3 (2015) 2962-2970.

[16] L.L. Hench, Bioceramics: from concept to clinic, J. Am. Ceram. Soc. 74 (1991) 1487-1510.

[17] A.A.A. Barros, A. Alves, C. Nunes, M.A. Coimbra, R.A. Pires, R.L. Reis, Carboxymethylation of ulvan and chitosan and their use as polymeric components of bone cements, Acta Biomater. 9 (2013) 9086-9097.

[18] F.O. Gomes, R.A. Pires, R.L. Reis, Aluminum-free glass-ionomer bone cements with enhanced bioactivity and biodegradability, Mater. Sci. Eng. C 33 (2013) 1361-1370.

[19] S.M. Rabiee, N. Nazparvar, M. Azizian, D. Vashaee, L. Tayebi, Effect of ion substitution on properties of bioactive glasses: a review, Ceram. Int. 41 (2015) 7241-7251.

[20] M.N. Rahaman, D.E. Day, B. Sonny Bal, Q. Fu, S.B. Jung, L.F. Bonewald, Bioactive glass in tissue engineering, Acta Biomater. 7 (2011) 2355-2373.

[21] H.B. Pan, X.L. Zhao, X. Zhang, K.B. Zhang, L.C. Li, Z.Y. Li, Strontium borate glass: potential biomaterial for bone regeneration, J. R. Soc. Interface 7 (2010) 1025 1031.

[22] W. Huang, D. Day, K. Kittiratanapiboon, M. Rahaman, Kinetics and mechanisms of the conversion of silicate (45S5), borate, and borosilicate glasses to hydroxyapatite in dilute phosphate solutions, J. Mater. Sci. Mater. Med. 17 (2006) 583-596.

[23] R.A. Pires, I. Abrahams, T.G. Nunes, G.E. Hawkes, Multinuclear magnetic resonance studies of borosilicate glasses for use in glass ionomer cements: incorporation of $\mathrm{CaO}$ and $\mathrm{Al}_{2} \mathrm{O}_{3}$, J. Mater. Chem. 16 (2006) 2364-2373.

[24] R.A. Pires, I. Abrahams, T.G. Nunes, G.E. Hawkes, The role of alumina in aluminoborosilicate glasses for use in glass-ionomer cements, J. Mater. Chem. 19 (2009) 3652-3660.

[25] H. Wang, S. Zhao, W. Xiao, J. Xue, Y. Shen, J. Zhou, Influence of Cu doping in borosilicate bioactive glass and the properties of its derived scaffolds, Mater. Sci. Eng. C 58 (2016) 194-203.

[26] J.S. Fernandes, M. Martins, N.M. Neves, M.H.F.V. Fernandes, R.L. Reis, R.A. Pires, Intrinsic antibacterial borosilicate glasses for bone tissue engineering applications, ACS Biomater. Sci. Eng. 2 (2016) 1143-1150.

[27] P.J. Marie, P. Ammann, G. Boivin, C. Rey, Mechanisms of action and therapeutic potential of strontium in bone, Calcif. Tissue Int. 69 (2001) 121-129.

[28] A. Hoppe, N.S. Güldal, A.R. Boccaccini, A review of the biological response to ionic dissolution products from bioactive glasses and glass-ceramics, Biomaterials 32 (2011) 2757-2774.

[29] S. Hesaraki, M. Gholami, S. Vazehrad, S. Shahrabi, The effect of Sr concentration on bioactivity and biocompatibility of sol-gel derived glasses based on $\mathrm{CaO}-$ $\mathrm{SrO}-\mathrm{SiO}_{2}-\mathrm{P}_{2} \mathrm{O}_{5}$ quaternary system, Mater. Sci. Eng. C 30 (2010) 383-390.

[30] C. Wu, W. Fan, M. Gelinsky, Y. Xiao, P. Simon, R. Schulze, Bioactive SrO-SiO glass with well-ordered mesopores: Characterization, physicochemistry and biological properties, Acta Biomater. 7 (2011) 1797-1806.

[31] M.E. Santocildes-Romero, A. Crawford, P.V. Hatton, R.L. Goodchild, I.M. Reaney, C.A. Miller, The osteogenic response of mesenchymal stromal cells to strontium-substituted bioactive glasses, J. Tissue Eng. Regener. Med. 9 (2015) 619-631.

[32] E. Gentleman, Y.C. Fredholm, G. Jell, N. Lotfibakhshaiesh, M.D. O’Donnell, R.G. Hill, The effects of strontium-substituted bioactive glasses on osteoblasts and osteoclasts in vitro, Biomaterials 31 (2010) 3949-3956.

[33] G. Brooke, M. Cook, C. Blair, R. Han, C. Heazlewood, B. Jones, Therapeutic applications of mesenchymal stromal cells, Semin. Cell Dev. Biol. 18 (2007) $846-858$.

[34] J.S. Fernandes, P. Gentile, R. Moorehead, A. Crawford, C.A. Miller, R.A. Pires, P.V. Hatton, R.L. Reis, Design and properties of novel substituted borosilicate bioactive glasses and their glass-ceramic derivatives, Cryst. Growth Des. 16 (2016) 3731-3740.

[35] C. Maniatopoulos, J. Sodek, A.H. Melcher, Bone formation in vitro by stromal cells obtained from bone marrow of young adult rats, Cell Tissue Res. 254 (1988) 317-330.
[36] K.J. Livak, T.D. Schmittgen, Analysis of relative gene expression data using realtime quantitative PCR and the 2- $\Delta \Delta C T$ method, Methods 25 (2001) 402-408.

[37] I. Rajzer, E. Menaszek, R. Kwiatkowski, W. Chrzanowski, Bioactive nanocomposite PLDL/nano-hydroxyapatite electrospun membranes for bone tissue engineering, J. Mater. Sci. Mater. Med. 25 (2014) 1239-1247.

[38] B.P. Chan, K.W. Leong, Scaffolding in tissue engineering: general approaches and tissue-specific considerations, Eur. Spine J. 17 (2008) 467-479.

[39] S.-L. Liang, W.D. Cook, G.A. Thouas, Q.-Z. Chen, The mechanical characteristics and in vitro biocompatibility of poly(glycerol sebacate)-Bioglass ${ }^{\circledR}$ elastomeric composites, Biomaterials 31 (2010) 8516-8529.

[40] V. Thomas, S. Jagani, K. Johnson, M.V. Jose, D.R. Dean, Y.K. Vohra, et al., Electrospun bioactive nanocomposite scaffolds of polycaprolactone and nanohydroxyapatite for bone tissue engineering, J. Nanosci. Nanotechnol. 6 (2006) 487-493.

[41] S.I. Jeong, E.K. Ko, J. Yum, C.H. Jung, Y.M. Lee, H. Shin, Nanofibrous poly(lactic acid)/hydroxyapatite composite scaffolds for guided tissue regeneration, Macromol. Biosci. 8 (2008) 328-338.

[42] A.K. Shakya, R. Holmdahl, K.S. Nandakumar, A. Kumar, Polymeric cryogels are biocompatible, and their biodegradation is independent of oxidative radicals, J. Biomed. Mater. Res. Part A 102 (2014) 3409-3418.

[43] G. Liao, S. Jiang, X. Xu, Y. Ke, Electrospun aligned PLLA/PCL/HA composite fibrous membranes and their in vitro degradation behaviors, Mater. Lett. 82 (2012) 159-162.

[44] M. Navarro, M.P. Ginebra, J.A. Planell, C.C. Barrias, M.A. Barbosa, In vitro degradation behavior of a novel bioresorbable composite material based on PLA and a soluble CaP glass, Acta Biomater. 1 (2005) 411-419.

[45] A. Zonari, S. Novikoff, N.R.P. Electo, N.M. Breyner, D.A. Gomes, A. Martins, Endothelial differentiation of human stem cells seeded onto electrospun polyhydroxybutyrate/polyhydroxybutyrate-co-hydroxyvalerate fiber mesh, PLoS One 7 (2012) e35422.

[46] S. Amorim, A. Martins, N.M. Neves, R.L. Reis, R.A. Pires, Hyaluronic acid/poly-Llysine bilayered silica nanoparticles enhance the osteogenic differentiation of human mesenchymal stem cells, J. Mater. Chem. B 2 (2014) 6939-6946.

[47] P.J. Marie, O. Fromigué, Osteogenic differentiation of human marrow-derived mesenchymal stem cells, Regener. Med. 1 (2006) 539-548.

[48] S. Peng, G. Zhou, K.D.K. Luk, K.M.C. Cheung, Z. Li, W.M. Lam, Strontium promotes osteogenic differentiation of mesenchymal stem cells through the Ras/MAPK signaling pathway, Cell. Physiol. Biochem. 23 (2009) 165-174.

[49] F. Yang, D. Yang, J. Tu, Q. Zheng, L. Cai, L. Wang, Strontium enhances osteogenic differentiation of mesenchymal stem cells and in vivo bone formation by activating Wnt/catenin signaling, Stem Cells 29 (2011) 981-991.

[50] H.-W. Kim, H.-H. Lee, J.C. Knowles, Electrospinning biomedical nanocomposite fibers of hydroxyapatite/poly(lactic acid) for bone regeneration, J. Biomed. Mater. Res. Part A 79A (2006) 643-649.

[51] A.M.S. Reis, L.G.R. Ribeiro, N.M. Ocarino, A.M. Goes, R. Serakides, Osteogenic potential of osteoblasts from neonatal rats born to mothers treated with caffeine throughout pregnancy, BMC Musculoskel. Dis. 16 (2015) 1-11.

[52] N.I. zur Nieden, G. Kempka, H.J. Ahr, In vitro differentiation of embryonic stem cells into mineralized osteoblasts, Differentiation 71 (2003) 18-27.

[53] M.C. Munisso, J.-H. Kang, M. Tsurufuji, T. Yamaoka, Cilomilast enhances osteoblast differentiation of mesenchymal stem cells and bone formation induced by bone morphogenetic protein 2, Biochimie 94 (2012) 2360-2365.

[54] G.R. Kirkham, S.H. Cartmell, Chapter 13: genes and proteins involved in the regulation of osteogenesis, in: N. Ashammakhi, R. Reis, E. Chiellini (Eds.), Topics in Tissue Engineering, 2007.

[55] L.A. Strobel, N. Hild, D. Mohn, W.J. Stark, A. Hoppe, U. Gbureck, Novel strontium-doped bioactive glass nanoparticles enhance proliferation and osteogenic differentiation of human bone marrow stromal cells, J. Nanopart. Res. 15 (2013) 1-9.

[56] Q. Tu, P. Valverde, J. Chen, Osterix enhances proliferation and osteogenic potential of bone marrow stromal cells, Biochem. Biophys. Res. Commun. 341 (2006) 1257-1265.

[57] J. Isaac, J. Nohra, J. Isaac, E. Jallot, J. Nedelec, A. Berdal, et al., Effects of strontium-doped bioactive glass on the differentiation of cultured osteogenic cells, Eur. Cells Mater. 21 (2011) 130-143. 